permanently derange the dynamic equilibrium of the alcohol in early stages of tuberculosis. The old-time nerve centers. Its repetition fixes this condition, and prescription to drink all the spirits possible when the power of resistance to disease becomes less. Local lesions of the lungs were made out, always ended inflammation from infectious agents, from injuries, either in precipitating the disease to more rapid death strains and drains, can not be overcome and death fol- or a low form of dementia and inebriety. The latter lows. Alcohol has been the factor which made these was always associated with delirium and exhaustion, conditions possible, and destroyed the vis medica and terminated in some acute disease.

naturce.

The following case represents a class which will

In this general outline of the facts concerning the power of alcohol as a factor in central nerve disease, the following facts may be restated:

1. Alcohol is a poison of the narcotic class, with a special tendency to act on the brain and nervous system.

2. In a general way the effects of alcohol are the same. Disturbing the functional processes and diminishing the sensory activities, ending in general mus. cular depression and mental enfeeblement.

3. A general psychical paralysis beginning with the ego and extending to all parts of the body, varying in some slight degree, but marked in all cases.

4. In dynamics of the brain there is a continuous gathering and discharge of nerve energy. This is increased, retarded and perverted by drugs, foods and environments.

5 . The change of this nerve energy is seen in the functional disturbances of the body. In early and late life it is very prominent.

6. Alcohol produces marked functional derangements. It both releases and concentrates nerve energy. The increased and diminished vascular actions, with diminished sensory power, point to profound disturbances of the dynamic forces of the brain.

7. Periodic drinking is a convulsive disease like epilepsy, and is of the same family group. General paralysis is another similar disease, associated with exhaustion.

8. Alcohol acts on the body psychologically, physiologically and pathologically, and is a literal paralysis and dissolution.

9. No other substance known in common use has such a profound destructive action on waste and repair of the body.

10. This opens the door for many diseases by destroying the power of resistance and enfeebling all the powers of life.

\title{
INEBRIETY AND TUBERCULOSIS, ALLIED DISEASES.
}

Presented to the Section on Neurology and Medical Jurisprudence at the Forty-eighth Annual Meeting of the American Medical Association held at Phlladelphia, Pa., June 1-4, 1897.

BY T. D. CROTHERS, M.D.

SUPERINTENDENT WALNUT LODGE HOSPITAL, HARTFORD, CONN.

The very close relationship between these diseases has been noticed for many years.

In certain families, tuberculosis and inebriety alternate. Some of the members will drink to great excess, then abstain, contract tuberculosis and die. Others will have all the symptoms of tuberculosis, begin to drink and recover from the consumption, become inebriates and die suddenly of acute pneumonia or nephritis. It is very commonly observed that inebriates who abstain from all use of spirits, calling themselves cured or reformed, soon contract tuberculosis and die. Such cases are very acute and rapid in their progress and termination. Anoth $ə$ r fact never understood was the apparently abortive influence of $/$ strain. 
Inebriety is simply narcotism to remove the pain and suffering from the defective functional activities.

Heredity has left the person with low and defective vitality, with feeble power of resistance and inability to adjust himself to the changing surroundings. $\mathrm{He}$ is in a state of chronic exhaustion, continually using up nerve energy that is replaced with great difficulty. His defective brain is unable to conserve force and hold it in reserve for emergency. Hence the seductive relief which comes from the narcotism of alcohol. Inebriety is only a symptom of this central bankruptcy of energy and power to develop force for the functional activities of life. The same conditions of weakness and degenerations diminish the power of resistance to the attacks of microbes and tuberculosis. Also the same conditions make the narcotism of alcohol fascinating and create the impression of strength by concealing the physical pain and demands for relief. In the latter case the degenerations go on, more profoundly effecting the higher centers with sensory hallucinations and delusions and changing the metabolism of the body. While alcohol apparently checks the invasion of the microbes and changes the symptoms, it creates new centers of degeneration and destroys the powers of resistance to every condition of strain and drain on the body. The removal of alcohol rouses the predisposition and favoring conditions of the soil for the growth of the bacillus.

If the degeneration has not taken on acute forms in other centers as in inflammation, tuberculosis may follow. A great variety of facts attest these observations and suggest a wide field for more exhaustive studies. One of these facts shows that families of inebriates and consumptives are often very fecund and numerous. Where the degeneration of the parents is unmistakable, the number of children born are often very large, confirming the oft noted fact, that just before the final extinction of the race a supreme effort is made to perpetuate the seed and save it from final extinction. The oak tree about to die will be over-burdened with acorns. The mongrel dog, whose race is nearly run out, will have an unusually large litter of puppies, which will die in infancy. While the families of inebriates are often very large they do not live long. If they live through childhood, they carry with them into maturity defects which soon end in extinction. Two degenerate members of a famous family in New York married. Both were moderate drinkers. Of the thirteen children born five died in infancy and eight grew to maturity. Three of this number died of tuberculosis, and were inebriates, one succumbed to acute pneumonia, one became demented, two died of some low form of fever associated with rheumatism. One is still living, but is feebleminded, and has been eccentric and partially demented all his life. All the decendants of these persons are dead.

Another typical illustration of this class came under my care. Three members of a family of nine came for treatment for inebriety. One relapsed and became insane; the second was killed by accident; the third is a low drunkard; two of the remaining children have died of tuberculosis; one is an eccentric reformer with extreme zeal and little wisdom; one is a paranoiac single woman. The parents of these children were wealthy, drinking persons, without business, who died in middle life from some acute disease.

These dying families are by no means uncommon, especially in the older sections of the cuuntry appear in the two extremes of either great fecundity or barrenness. In one case a sudden large progeny will follow, in the other barrenness, and in both profound constitutional diseases appear with tuberculosis, inebriety and insanity in its obscure and modified forms. Where a general history indicates that the family is degenerating, growing weaker in appearance and conduct retrograding, tuberculosis and inebriety with hysteria, eccentricity, rheumatism, and a variety of nerve disorders are almost certain to follow. Inebriety and various forms of drug addictions come first, then tuberculosis or acute affections such as pneumonia, hepatitis, nephritis and affections of the heart. These facts are not observed carefully because often the person or victim manifests some unusual form of vigor and ability before these diseases appear. A son of one of these dying families took high honors at college and entered professional life with brilliant prospects. $\mathrm{He}_{\theta}$ attained eminence, suddenly became an inebriate and two years later died of tuberculosis. His inherited degeneration was overlooked in his precocious brilliancy, and display of vigor masked the early dissolution which concentrated in inebriety and tuberculosis. Not all these cases become tuberoulous or inebriates; other organic affections appear. The heart and kidneys suffer with the stomach, and a great variety of nervous affections which are likely to concentrate on the lungs, or depress the nerve centers to such a degree as to demand narcotics for relief. This sudden or gradual lowering of the co-ordinating nerve centers in vigor and power may be felt first in the lungs, and then the microbe's soil is ready for the growth of tuberculosis. The inheritance of low vitality and a predisposition to seek relief in spirits and drugs that cover up and relieve this pain and physical unrest, still farther lowers the nerve centers of co-ordination, literally enfeebling the power of resistance to all microbic invasions, and making the possibility of tuberculosis more certain.

The neurotic origin of tuberculosis has been for many years urged by Dr. Mays of Philadelphia, in many able studies. He has shown conclusively that tuberculosis is far more common in feeble-minded neurotic families. He has proven that it follows more frequently in families of inebriates and those who are eccentric, hysteric and possess a neuro-psychopathic constitution. These facts are not new but have been observed by many competent authorities for years. Dr. Mays has restated them with much additional evidence and many new conclusions that are unques. tionable, proving the neurotic origin of consurnption.

The present great precautions used to prevent the transmission of tuberculosis germs overlook this fact. The common neurotic origin of tuberculosis and inebriety is also noted in the paroxysmal character of their progress and termination. Cases of tuberculosis come on suddenly, pass rapidly to a fatal termination, or are marked by long irregular halts and apparent recoveries, which are supposed to be due to certain remedies or means used. These halts are paroxysmal, irregular and followed by other diseases or death from the original disease. Climate cures, drug cures, food cures, are all secondary to nerve rest and hygienic measures which build up the brain and central nervous system.

Inebriety begins the same way, in many cases suddenly, and is followed by halts and paroxysmal changes. Then the drink crave breaks out again, subsides, then They changes to acute inflammation of the lungs or kidneys. 
A period of moderate drinking seems to prepare the nervous system for drink-storms and excesses.

In tuberculosis, bronchial catarrhs and irritations of the throat, lead up in the same way to pronouncer tuberculosis. In many cases of tuberculosis a marked mania exists for drugs of some kind. This is the same craving for relief that possesses the inebriate. The former is filled with the hope of virtue in drugs, is credulous, expectant, ever ready to try every new thing, never doubting its possible good effects. Filled with delusions of strength and final cure of his malady, the inebriate has the same boundless confidence in his power to abstain and ability to use spirits at all times and recover from the effects. The similarity of these cases can be traced in a great variety of symptoms which are identical in both cases. In both of these diseases there are similar nerve degenerations which may concentrate on any organ of the body.

Dr. Alison, in an exhaustive paper on the etiology of cirrhosis and pulmonary phthisis among inebriates, found phthisis more common among those of active habits.

He studied eighteen inebriates in fifty-four cases of phthisis, and asserted (Archives Générales de Mélecine, Paris), as his positive conclusion that the use of alcohol predisposed to consumption by lowering the powers of nerve resistance, and by creating a constant source of irritation in the bronchi and lungs from the elimination of the alcohol. He was also clear in his conviction that inebriety and consumption were interchangeable, one following the other from the same general causes and both more likely to occur before 40. After that, acute inflammations followed more readily.

Dr. Mays, in the Journal of Inebriety for 1889 , reported a number of cases from German authorities where death occurred from some form of inebriety, and the postmortem revealed tuberculosis and lung degeneration. In the same paper he reports a number of cases of inebriety following in the children of consumptives, and of phthisis or consumption appearing in children of inebriate parents. Dr. Haycraft, in "Darwinism and Race Progress," declares that criminals, inebriates, lunatics and consumptives, are all born with neuro-psychopathic constitutions, and in no other affection does heredity play so important a part.

Dr. Irwell, in "Racial Deterioration," describes the neuro-psychopathic constitution as a permanent condition of defect and weakness of the psychical nerve centers, noted in instability of nerve and brain power, lack of persistency, headaches, insomnia, indigestion, great susceptibility to environments, exaggerated emotional activities and a general insane temperament.

Dr. Williams, in his work on pulmonary consumption, written some years ago, describes the close relation between inebriety and consumption, calling them members of the same family group of diseases, one alternating with the other.

The fact has been noted by many specialists that inebriety often terminates in acute inflammation of the lungs and kidneys, coming on suddenly, showing that exhaustion has a special tendency to concentrate in this way.

Dr. Clouston of Edinburgh, in a late report says, that it is surprising how often insanity, consumption and inebriety appear in the same family and follow down to extinction among the descendants.

When one of these diseases is present the others are most likely to follow. Dr. Payne, in his "Pathology of Chronic Alcoholism" says: "I can find no evidence to support the opinion that the free use of alcohol checks the progress of tuberculosis." On the contrary, the impression seems well founded and firmly held by many authorities that inebriety follows tuberculosis, and that they alternate one with the other frequently; at least there can be no question that they are very frequently associated.

Dr. Sharkey, in the London Pathological Society, claims that disturbances and lesions of the vagus nerve by lowering the nutrition of the lung, predispose it to become the nidus of the bacillus of tuberculosis. Hence all inebriates are predisposed to tuberculosis, and are more likely to contract this disease than any others.

The evidence of the neurotic origin of tuberculosis is often overlooked in bacterial study. The same extraordinary care to prevent infection in public places should extend farther back to heredity, alcohol and the great strains and drains that lower the vigor and lessen the power of resistance and make it possible for bacteria to find favorable soil for the destruction of the body.

\section{THE INFLUENCE OF HYPNOTIC SUGGES- TION UPON PHYSIOLOGIC PROCESSES.}

Presented to the Section on Neurology and Medical Jurisprudence, at the Forty-eighth Annual Meeting of the American Medical Association at Philadelphia, Pa., June 1-4, 1897 .

BY R. OSGOOD MASON, A.M., M.D. NEW YORK, N. Y.

That hypnotism is a fact, and that the power of suggestion is marvelously increased while the patient or subject is in the hypnotic condition, are now truisms to all who have been students in that domain of psychology to which these subjects belong. The old rubbish of superstition, supernaturalism, and even of occultism, is in part at least swept away, and these two facts now stand out clear and distinct. It remains, by observation and experiment, to test their possibilities and ascertain to some degree the limit of their influence and usefulness.

Permit me to remark regarding the general drift of therapeutic practice in nervous diseases no less than in other departments of clinical work, that notwith. standing the tendency to specialization, and the multiplication of remedies in each special department, still amongst the wisest and most conservative in all directions the tendency is toward moderation and simplicity in the use of remedies; and thought is given especially to the repair of organs through physiologic processes, rather than the search for authoritatively endorsed specifics. Nothing can better illustrate my meaning than the treatment of epilepsy during the past thirty or forty years; there were first the salts of silver, then the salts of zinc, then the bromid of potash, and then that remarkable shotgun invented by Brown-Séquard, in which all the known bromids were combined; later the bromid of sodium alone was the favorite, and now the general feeling is that in the ultimate results bromids are harmful, and that diet, regimen, air, and regulated exercise are much the most important factors in the treatment of this formidable disease. In other words, attention is being directed from specifics to the consideration of what can best be done for the repair and healthy action of damaged nerve cells and imperfectly functioning organs. 